\title{
La teoria della lettura
}

\author{
Vittorio Spinazzola
}

Una riflessione teorica sui problemi della lettura chiede anzitutto di enunciare alcuni presupposti concettuali. Il primo è che il leggere sia una forma di attività mentale basata sempre su un interesse utilitario: nel senso che se io leggo un libro, lo faccio perché penso di trarne un vantaggio, un arricchimento della mia vita interiore, che mi ripaghi del tempo e delle energie spese leggendo. Il secondo è che la scelta del tipo di lettura da fare dipende da me, dalle mie intenzioni personali, cioè dallo scopo specifico che mi propongo di conseguire: posso aver in mente di appagare un desiderio di svago, di rilassamento psichico, oppure di ottenere un ammaestramento morale e conoscitivo, oppure di procurarmi delle informazioni o ancora, ed è il caso più rilevante, di soddisfare delle esigenze di tipo estetico-letterario.

Quanto a coloro che compiono l'atto di leggere, possiamo ricorrere a una distinzione categoriale tra il lettore come soggetto singolo e il pubblico come soggetto collettivo. Il secondo termine indica l'insieme delle persone che si dedicano a certe letture in un ambiente storicamente e culturalmente determinato, rifacendosi a criteri di comportamento omogenei. Il primo termine invece si riferisce all'io leggente, come individuo dotato di una sua fisionomia irripetibile e quindi portato a reagire in maniera propria, autonoma, agli stimoli, alle proposte che gli vengono dal mondo della scrittura.

Queste considerazioni generalissime vogliono delineare la prospettiva di una teoria critica della lettura che abbia un fondamento di realtà storico-antropologica, in quanto impostata sulla persuasione che l'atto di leggere si strutturi sempre secondo gli stessi paradigmi, dovunque si sia insediata la civiltà del libro moderna. La mia ipotesi di lavoro è che la tipologia delle risorse psichiche mobilitate dalla lettura non cambi con il variare delle condizioni ambientali. Non solo, ma secondo me il lettore colto non legge in modo costitutivamente diverso dal lettore incolto: anche se, naturalmente, applica i procedimenti della lettura in maniera più consapevole e complesssa.

Abbiamo così a disposizione alcune premesse utili per cercar di risolvere qualche equivoco ed eliminare vari luoghi comuni che gravano sullo studio di un insieme di argomenti non ancora esplorati adeguatamente in tutta la loro portata istituzionale. Se ci si pensa, la cosa è paradossale: noi sappiamo molto di più sulle operazioni dello scrivere che non su quelle del leggere. Eppure, la lettura è un'esperienza che coinvolge tanta più gente della scrittura; i lettori 
sono sempre stati ben più numerosi degli scrittori. Ma resta il fatto che chi legge si preoccupa poco di capire, di riflettere su quello che sta facendo, mentre si preoccupa invece molto di capire che cosa ha voluto fare l'autore del testo al quale sta dedicando la sua attenzione.

Come si sa, l'interesse per la fenomenologia e la metodologia del leggere è di data recente. Un punto di riferimento emblematico può esser indicato nel Qu'est que la litterature? di Sartre, anno 1947, dove il filosofo esistenzialista sostiene che senza lettori non c'è letteratura: un libro, se non ha nessuno che concretamente lo legga, resta solo un oggetto materiale privo di significato e di valore, un semplice insieme di fogli inchiostrati. Questa affermazione famosa merita sempre di essere ricordata perchè sintetizza la necessità di passare da una concezione ontologica della produzione letteraria a una concezione funzionalista e relazionale: si scrive per essere letti; tra il leggere e lo scrivere esiste un vincolo di interdipendenza; lo scrittore è sì il protagonista ma il lettore ha un ruolo coprotagonistico nelle dinamiche della letterarietà.

Nel corso del secolo, prima e soprattutto dopo Sartre, allo sviluppo delle indagini sui problemi della lettura hanno contribuito studiosi di formazione e provenienza disciplinare molto diversa: dalla linguistica alla psicopedagogia, dalla storia della letteratura a quella del libro, dalla teoria della comunicazione all'estetica filosofica alla sociologia. Tra gli italiani, impossibile non citare, sia pure alla rinfusa, almeno i nomi di Umberto Eco, Cesare Segre, Maria Corti, Armando Petrucci, Giuseppe Petronio, Graziella Pagliano, Franco Brioschi. La spinta decisiva per porre la questione lettura all'ordine del giorno è tuttavia quella impressa dalla cosiddetta scuola di Costanza, a partire dal 1967, quando Hans Robert Jauss pubblica Literaturgeschichte als Provokation der Literaturwissenschaft. È per merito dello stesso Jauss e dei suoi colleghi, anzitutto Wolfgang Iser, che ha preso davvero avvio un discorso sistematico di teoria della ricezione in campo letterario.

Piuttosto che compiere una rassegna analitica delle posizioni più significative oggi in campo, mi sembra però stimolante intervenire sinteticamente su alcuni nodi problematici non abbastanza posti in chiaro nel dibattito odierno delle idee. In effetti, una perplessità di fondo può essere suscitata dalle definizioni più autorevoli del ruolo del lettore, e delle procedure di lettura, anche e proprio nel pensiero dei teorici tedeschi. La figura di colui che legge appare concepita come una entità funzionale interna al testo, sprovvista di una autentica autonomia: la sua attività sembra dover consistere nell'adempiere le istruzioni in codice che l'opera scritta gli trasmette, in modo da far funzionare al meglio la macchina testuale, secondo il progetto di chi l'ha concepita. Orientamenti simili hanno il pregio indiscutibile di contrastare frontalmente la tesi, del tutto insostenibile, di chi esalta invece la libertà assoluta del lettore, ritenuto arbitro di reinventare il testo, senza preoccuparsi degli scopi e dei criteri secondo cui è stato realizzato, cioè facendone un puro pretesto per lo sviluppo della propria vita interiore. Ma il punto è che restiamo sempre nell'ambito di un modellistica concepita in chiave di intellettualismo elitario, con un forte connotato castale. 
In entrambi i casi, difatti, il lettore di cui si parla ha una configurazione astratta, virtuale: è il lettore più bravo che possa esserci, perfettamente in grado di padroneggiare le difficoltà presentate dai testi più esoterici: una specie di superuomo della lettura. In realtà poi, questa immagine ideale ha un contenuto concreto ben determinato: si tratta del letterato professionista, appartenente a pieno titolo alla casta dei dotti. Implicitamente o esplicitamente, viene dato per certo che solo di costui vale la pena di occuparsi, analizzandone le reazioni di fronte alla pagina scritta. Quanto al lettore medio e comune, è considerato inesistente sul piano letterario e quindi indegno di venire assunto come oggetto di riflessione teorica. I suoi comportamenti sono confinati pregiudizialmente nell'ambito di un conformismo anonimo e amorfo: tutt'al più, li si potrà esaminare in sede di indagini empiriche, inchieste sociologiche o ricerche di mercato.

In questo modo però viene eluso il problema decisivo, che consiste proprio nel definire le costanti normative dell'attività di lettura, come viene esplicata da chiunque legga libri. La qualifica di lettore spetta infatti a ogni persona che decida liberamente di accostarsi a un testo scritto, col proposito di ricavare un vantaggio da questa esperienza. Solo così è possibile chiarire la natura istituzionale della lettura, riconoscendo i fattori di invarianza che soggiacciono alla fenomenologia infinita degli atti compiuti da qualsiasi io leggente, al di là del tipo di pubblico cui appartiene e di tutte le differenze di status, di competenza, di gusto.

Ho già osservato all'inizio che il pubblico va inteso come il soggetto collettivo al quale lo scrittore si rivolge, consapevolmente o inconsapevolmente, nel dare corpo al suo lavoro. Per un lato, questo insieme di destinatari è suscitato dall'apparizione dell'opera: prima che venisse, appunto, pubblicata, essi sono soltanto una potenzialità genetica indeterminata. Per l'altro verso però, hanno presieduto alla sua genesi, perché nessun testo letterario nasce se non dal desiderio dell'autore di essere letto da qualcuno.

Il pubblico ha dunque una identità psicosociale obbiettiva, esterna a l'opera, ed è portatore non solo di attese ma di esigenze e domande circostanziate. È con queste richieste di lettura che l'autore si confronta: per accettarle o ricusarle, oltrepassarle o ribadirle. Certo, chi scrive pensa o può pensar di rivolgersi a un destinatario universale: ma in concreto non può non assumere a proprio interlocutore elettivo un dato gruppo o strato o settore di utenza, sulla cui disponibilità ritiene di fare affidamento. Ed è l'immagine di questo specifico fruitore, colto o incolto, spregiudicato o conformista, avanzato o retrivo, che noi possiamo scorgere in filigrana nell'organismo testuale.

Il pubblico insomma è un'entità che va sempre declinata al plurale. La sua varietà di articolazioni consente di prospettare le vicende letterarie come una dinamica di incontro tra molteplici domande e offerte concorrenti. Naturalmente, la correlazione tra atti di scrittura e atti di lettura è asimmetri$\mathrm{ca}$, perché la responsabilità dello scrivere è ben diversa da quella del leggere: si legge soltanto ciò che sia stato scritto. Ciò non toglie che i processi della lettura rispondano specularmente a quelli della scrittura: affermazione incontrover- 
tibile, quando si sia d'accordo sul fatto che il lettore costituisce una polarità dialogica nei riguardi dell'autore.

Ora, il punto è che chiunque legga qualcosa, rivendica il suo diritto non solo ad approvare ma eventualmente a disapprovare ciò che lo scrittore gli offre. E questo significa prendere posizione, facendo valere le proprie istanze. Leggere vuol dire esaminare un testo, per vedere se corrisponde alla fiducia che abbiamo concesso al suo autore quando lo abbiamo prescelto. Certo, occorre fare i conti con l'oggettività del prodotto scritto; ma per appropriarselo davvero, bisogna pure riportarlo ai nostri interessi e intenzioni, attitudini e orientamenti soggettivi. Ciò lascia spazio a ogni possibilità di fraintendimento, di equivoco, di errore. Ma la condizione di efficacia della lettura sta proprio in questo rischio. Nessun atto fruitivo risulta mai proficuo se non si fonda su una libertà operativa, da parte di chi legge: cioè sulla possibilità di criticare quel che viene letto.

Queste considerazioni sommarie vogliono chiarire perché al concetto superindividuale di pubblico vada appaiato quello di io leggente, per designare la persona singola, nella sua fisionomia storicamente condizionata ma esistenzialmente irriducibile. Di qui viene la natura particolare delle sue facoltà e inclinazioni, dei propositi e dei criteri con i quali si dà al leggere. Nessun leggente è mai uguale a un altro; anzi, nessuna lettura è mai equiparabile a un'altra, nemmeno fra quelle compiute dallo stesso soggetto, proprio perché ciascuna porta delle modifiche alla sua identità: altrimenti, del resto, perché varrebbe la pena di continuar a leggere o rileggere libri?

Siamo a un nodo davvero centrale: non basta discorrere sul come si legge, bisogna spiegare perché si legge. E questa è una domanda che inequivocabilmente riguarda non solo il lettore letterato ma anche il più ingenuo, il meno esigente. Appunto perciò la risposta non può non avere una latitudine massima, ponendosi sul piano delle costanti metastoriche, delle disposizioni iscritte nel nostro codice biopsichico. Torniamo allora ai due concetti di interesse e intenzione di lettura.

Se io mi accingo a leggere un libro, lo faccio perché penso che mi convenga farlo: me ne attendo una qualche sorta di gratificazione. Quel dato libro mi pare interessante, nel senso che mi fa provare interesse all'idea di leggerlo. I motivi per cui sono portato a sceglierlo possono essere dei tipi più diversi, e possono risentire delle influenze, dei condizionamenti esterni più svariati. Ma in ogni caso io sono mosso dall'esigenza di colmare una mia lacuna, di soddisfare un mio bisogno, insomma dalla volontà di migliorare, illuminare, compensare con l'esperienza mentale della lettura la mia esperienza pratica di vita.

La decisione di accostarmi proprio a quel dato libro invece che a un altro può anche essere il risultato di una pluralità di motivi, consapevoli e inconsapevoli. C'è però sempre una istanza catalizzatrice, che guida la scelta dell'opera ritenuta più conveniente, e assieme del modo di leggerla più vantaggioso. L'opzione di lettura si svolge insomma sulla base di una intenzione finalizzata, che circoscrive il campo delle preferenze possibili e che può essere strutturata 
più o meno limpidamente ma comunque prefigura l'orientamento del lettore rispetto al testo.

La particolarità dell'intenzione di lettura letteraria consiste nel proposito di appagare i desideri del nostro immaginario estetico, servendoci di un prodotto scritto elaborato proprio a questo scopo. Il calcolo da cui sono mosso è quello di acquistare un bene che mi sia utile per esaudire le richieste di esteticità che sorgono dalla mia vita interiore. Naturalmente, nell'effettuare la mia scelta io cercherò di indirizzarmi su testi adeguati alle mie aspettative, cioè che non si collochino né troppo al di sopra né troppo al di sotto delle capacità di lettura delle quali dispongo. Ma questa non può non essere cosa ovvia.

Viene dunque posta in essere una tipica situazione di scambio, che mette in rapporto due parti contraenti: il lettore rappresenta la domanda, lo scrittore impersona l'offerta. Oggetto della contrattazione è il testo, nella sua materialità di merce libraria. Come qualsiasi evento di scambio, anche questo può rivelarsi più o meno proficuo per l'acquirente. Quando chi legge si sente accontentato dalla lettura, remunera l'autore con un riconoscimento di merito, che poco o tanto contribuisce al suo successo e alla sua fama. Non è detto però che le cose vadano sempre bene. Sembra banale ricordarlo, eppure si tratta di una circostanza che non ha mai sollevato un'attenzione adeguata in sede di teoria della ricezione: del resto, lo si capisce, giacché il lettore al quale i teorici preferiscono riferirsi è così dotto e astuto da far supporre che non effettuerà mai delle scelte sbagliate, e leggerà solo testi dei quali valga la pena di occuparsi. Nondimeno, tutti sanno che invece può capitare, altroché, di trovarsi delusi al termine della lettura: e di concludere che si è fatto un cattivo affare impegnandocisi.

Nella sua concretezza, questa terminologia economicistica mette in rilievo un dato fondamentale: la lettura richiede un dispendio di tempo e di energie, che costituiscono il prezzo da pagare per impadronirsi del prodotto scritto, assimilandone mentalmente le qualità. E questo prezzo potrà apparire equo oppure sproporzionato, agli occhi di chi lo esborsi, secondo l'utilità dell'acquisto. Nel caso della lettura letteraria, l'utilità si misura in termini di godimento estetico. Siamo a un'altra questione capitale, per lo più trattata in modo insoddisfacente dalla teoria della ricezione.

Il piacere della lettura non può esser fatto risiedere esclusivamente nella dimensione ermeneutica, ossia nell'impresa di decifrazione della significanza testuale. Conta anche, eccome, l'apprezzamento o il deprezzamento di ciò che si sta leggendo, interpretando, decrittando. Capire un'opera significa coestensivamente valutarla; l'atto di leggere coincide in ogni suo momento con l'atto di giudicare. E la lettura non può non concludersi con una stesura di bilancio, tra quanto ci è costato compierla e quanto se ne è ottenuto.

Detto in altre parole, l'esperienza di leggere si risolve in un raffronto di prezzi e ricavi: quanto mi ha reso la lettura di quel testo, in confronto all'impegno che ho dovuto profondere per appropriarmene? Se si tratta di una lettura letteraria, l'interrogativo suona così: quale soddisfazione del mio immaginario mi ha procurato? La risposta implica immancabilmente la formulazione di 
un verdetto, ossia l'attribuzione di un segno di valore o disvalore al testo in causa. Se il saldo è all'attivo, quel testo valeva la pena di leggerlo: il rischio affrontato quando lo si è scelto è stato superato positivamente, le energie psichiche consumate per leggerlo sono state spese bene: si tratta di un prodotto degno di venir annesso al patrimonio di buone letture depositato nella memoria del lettore. Altrimenti, il libro sarà da buttare nel dimenticatoio.

Questa prospettiva teorica ha il vantaggio di spostare l'accento dalla questione tradizionale del valore estetico, astratta e irrisolvibile, alla concretezza dei processi di valorizzazione che investono certi prodotti a scapito di altri, su scenari storici in continua evoluzione. Così affermava già nel 1936 Jan Mukarôvský, uno fra i membri più illustri del Circolo di Praga. Alla valutazione sociale dei testi letterari concorrono tutti i tipi di pubblico che si affiancano e succedono nello spazio della letterarietà. E ovvio che l'efficacia maggiore spetti alle opinioni formulate dalle élites colte, il cui nucleo è formato dagli esperti professionistici della lettura. Ma ciò non contrasta con il fatto che ogni lettore esercita una sua funzione critica, diretta o indiretta, sui prodotti circolanti nella comunità cui appartiene: ognuno dunque collabora, come può e come sa, in positivo o in negativo, a determinare molecolarmente il sistema gerarchico dei valori letterari.

Essenziale è infatti ribadire che chiunque legga, per il fatto stesso di leggere, si arroga la facoltà di emettere un proprio personale giudizio sulle qualità buone o cattive del libro che ha letto, confermando o magari smentendo la fortuna di cui gode. Potrà trattarsi di un parere ragionato oppure di una semplice impressione frettolosa, non importa; ciò che conta è la rivendicazione di identità dell'io leggente, attuata esercitando il suo diritto insindacabile di valutare la funzionalità del prodotto scritto rispetto alle esigenze che avevano indotto a leggerlo. Nessuna autorità può togliere nemmeno al più umile lettore il potere di dichiararsi insoddisfatto di un'opera che non lo abbia convinto, non gli sia piaciuta.

Resta inteso che il criterio valutativo di un pubblico vasto ma culturalmente subalterno conta meno di quello della cerchia ristretta dei letterati, che sono coloro i quali decidono i canoni di lettura approvati istituzionalmente e compilano gli elenchi degli scrittori da includere nelle storie letterarie. È lapalissiano che le cose debbano andare così. Meno giusto, anzi profondamente sbagliato, è però che non si ritengano degne di considerazione storica e teorica le opere che ottengono il consenso più ampio da parte di un'utenza non qualificata.

Tutti gli interessi di lettura vanno considerati legittimi. E' un bieco pregiudizio aristocratico ritenere che la letteratura sia fatta solo delle opere che hanno il beneplacito dei letterati. Anche i prodotti meno raffinati, quando si dimostrino in sintonia profonda con l'immaginario dei lettori meno colti, svolgono una funzione letteraria: e con ciò stesso hanno diritto di essere inclusi nei domini della letterarietà: al posto che loro spetta, s'intende.

Del resto, oggi non dovrebbe più essere difficile ammettere che la produzione di intrattenimento o di consumo o di massa, come si preferisce chia- 
marla, non si costituisce solo per effetto della degradazione e imbastardimento di modelli letterari calati dall'alto verso il basso. Esistono anche fenomeni di senso contrario: anzi, proprio al livello delle pratiche di lettura e di scrittura meno istituzionalizzate possono verificarsi le spinte innovative destinate a ristrutturare con maggior forza il sistema della letterarietà ufficiale. La massima che il pubblico ha sempre ragione è ovviamente balorda: ma non lo è meno il parere che abbia sempre torto. La forma romanzo ha una nascita bassa, e così il fumetto, per non dire del cinema.

Una teoria della lettura che sviluppi con rigore i suoi postulati non può non invitare a un cambiamento radicale dell'idea di letteratura vigente, e quindi della metodologia da adibire alla ricerca applicata. L'ambizione ultima è di indole totalizzante: ricostruire il panorama della vita letteraria di una data epoca e paese nella sua interezza di articolazioni e stratificazioni, prendendo in esame tutto ciò che abbia avuto un successo di lettura: senza lasciarsi andare a nessuna indulgenza corriva, ma nemmeno ostentando un disprezzo snobistico.

Stiamo però passando dal campo della teoria a quello della storia della letteratura, e della lettura. Meglio non inoltrarcisi. Vale piuttosto la pena di concludere queste note confutando o almeno revocando in dubbio un'altra convinzione assiomatica molto diffusa: cioè che la miglior competenza basti a fare il miglior lettore. Non è vero. Tutti sanno che uno scrittore può essere espertissimo, può sciorinare le squisitezze di stile più eleganti eppure non riuscir a combinare un buon libro: ben che vada, farà della bella letteratura, in chiave manieristica e calligrafica. L'importanza di un'opera non si misura sulla capacità di maneggiare signorilmente gli strumenti del mestiere.

Allo stesso modo, per leggere bene, ossia produttivamente, non basta essere forniti di una cultura ampia e solida: tutt'al più, si arriverà a una buona lettura scolastica. Nell'analizzare un testo si può impiegare la dottrina più erudita, si può ricorrere ai criteri scientifici più raffinati, eppure non riuscir a cogliere l'essenziale, ciò che fa l'originalità dell'autore su cui si lavora. Non è detto insomma che un professore di belle lettere sia davvero sempre un interprete adeguato dei prodotti letterari. Del resto, nessun vero scrittore ha mai pensato di rivolgersi esclusivamente alla categoria dei professori.

Naturalmente, con questo non si vuol dire che la competenza di lettura non vada educata, ci mancherebbe altro. Anzi, va formata al meglio, con molta più intelligenza di quanto per lo più non accada a scuola. Il punto è che la competenza rappresenta una condizione necessaria ma non sufficiente per godere davvero del rapporto con il testo. Va rifiutata la boria dei dotti, persuasi che il lettore medio e comune non sia mai in grado di accostarsi ai testi in modo soddisfacente, perché il suo modo di leggere sarebbe sempre e solo improntato ai criteri più prevedibili e retrogradi.

Bisogna invece ribadire che anche il lettore più modesto può personalizzare la sua lettura, come le risorse del suo temperamento glielo suggeriscono, senza affatto mostrarsi di facile contentatura. La sensibilità e il gusto non sono appannaggio esclusivo dei letterati. Una competenza scarsa o poco addestrata 
è un limite evidente a capire e interpretare i testi più sofisticati. Ma sui libri alla sua portata, rispetto ai quali si senta competente, il lettore illetterato può dare prova di una spregiudicatezza indiscutibile; mentre magari il pubblico benpensante si limita a ossequiare i testi più canonici, o più alla moda, senza azzardarsi mai a contestarne il prestigio. 